\title{
Evaluación de conocimientos y actitudes sobre neuromitos en futuros/as maestros/as
}

\author{
Evaluation of neuromyth's knowledge and attitudes in future teachers
}

\author{
Adela Fuentes y Alicia Risso \\ Dpto. de Psicología, Universidade da Coruña (España)
}

\begin{abstract}
Resumen
En este trabajo se analizan la persistencia de neuromitos en futuros maestros estudiantes de la UDC, y sus actitudes respecto a aspectos relacionados con este mismo tópico. Se reflejan también los resultados de análisis comparativos con aquellos estudios de investigaciones precedentes recogidos en docentes de distintos ámbitos geográficos. Se concluye, a través del proceso de análisis de datos, que el nivel de formación de los futuros maestros podría perfilarse como una influencia favorable al proceso de disipación de neuromitos, y que se reflejaría, de igual modo, en sus actitudes.

Palabras clave: neuromitos, actitudes de estudiantes de magisterio, nivel de conocimientos
\end{abstract}

\begin{abstract}
In this paper prevalence of neuromyths among UDC future teachers, and their attitudes about same topic had been analyzed. Through a comparative analysis with results of previous research gathered in teachers from different geographical areas, we could conclude that the level of training of future teachers would emerge as a favorable influence to dispelling neuromyths. This fact would be reflected too in their attitudes.

Keywords: neuromyths, future teacher's attitudes, level of knowledge.
\end{abstract}

La incursión de las investigaciones neurocientíficas en el terreno de lo educativo, continúa siendo producto de un intenso debate debido al proceso de comercialización que se ha originado a partir de las mismas. Este fenómeno ha dado forma a un mercado en el que se ofertan desde juegos de ordenador que prometen producir un efecto gimnástico en el cerebro, hasta fórmulas casi mágicas para incrementar las capacidades cognitivas de los más pequeños, asegurando, de este modo, mejores niveles de rendimiento académico. Es en este contexto de proliferación de información respecto a lo que la neurociencia puede o no puede aportar a los procesos de optimización educativa donde surge el constructo de los neuromitos, articulado de modo que posibilite la ejemplificación de aquellos núcleos temáticos a través de los que se han ido originando un mayor número de creencias erróneas que promulgan una sinergia entre los estudios del cerebro y la educación escolar. El término neuromito fue acuñado por un neurocirujano, Alan Crockard, durante la década de los 80 , aludiendo con él a un tipo engañoso de saber sobre el cerebro dentro de la cultura médica. En el contexto educativo, los neuromitos fueron introducidos por la OECD en 2002, que dedicó un capítulo del informe Understanding The Brain. The Birth of a New Learning Science a este tópico: surge así la primera definición de neuromitos educativos, concebidos como concepciones erróneas en relación con el aprendizaje escolar, y modelándose como el reflejo del establecimiento de puentes sin fundamento entre los resultados de las investigaciones procedentes de las ciencias cognitivas, y la educación. Desde hace varias décadas, los neuromitos persisten en las escuelas, siendo utilizados, a menudo, para justificar enfoques de enseñanza ineficaces. En los años recientes las comunicaciones científicas respecto a este tópico se están incrementando, perfilando para el futuro un nuevo campo de investigación orientado a la construcción de una estructura que posibilite una mejor información de estas comunicaciones. Existen diversos núcleos temáticos a través de los que han indo emergiendo este tipo creencias, $\mathrm{y}$ que van a ir perfilando los principales neuromitos objeto de estudio en investigaciones precedentes. Entre los neuromitos estudiados para analizar el nivel de profusión entre los profesionales educativos se encuentran los recogidos por la $\operatorname{OCDE}(2002 ; 2007)$, referidos a creencias originadas en torno a los núcleos temáticos de la neuroplasticidad, los estilos de aprendizaje, la dominancia hemisférica o los períodos críticos. Respecto a las creencias erróneas analizadas entre los docentes Dekker, Lee, Howard-Jones y Jelle (2012), indican al respecto que en el neuromito de los estilos de aprendizaje subyace una base científica comprobada empíricamente que establece que los distintos tipos de información sensorial, (visual, auditiva y kinestésica) son procesados por áreas diferenciadas en el cerebro. De este hecho contrastado han ido emergiendo propuestas pedagógicas basadas en la identificación de la modalidad de preferencia de los estudiantes, infiriendo que la presentación de la información en la vía sensorial identificada, revertiría en mejoras del aprendizaje. Por otra parte, Calvert, Campbell, y Brammer (2000) apuntan que en comparación con los inputs unimodales, los inputs multimodales, semántica y/o espacialmente congruentes aumentarían la velocidad de discriminación produciendo mejoras en los tiempos de reacción. Geake (2008), por su 
parte, observa que la evidencia científica sitúa a los métodos pedagógicos que apoyan la presentación multisensorial como aquellos más adecuados para la optimización del aprendizaje: el procesamiento bimodal de información congruente presenta efectos supraaditivos (ver y escuchar al mismo tiempo la misma información proporciona mejores resultados que primero procesar visualmente y después auditivamente).

La OECD (2002), respecto a otro de los neuromitos analizados en cuanto a su prevalencia entre el colectivo docente, vino a subrayar la amplitud de extensión que alcanzó la creencia entre los educadores de un uso de sólo el $10 \%$ del cerebro. Geake (2008) apunta a que esta creencia eclosiona entre los educadores de finales del siglo XX reflejando las expectativas de los docentes respecto a aquellas capacidades desaprovechadas por los estudiantes, pero que supone una afirmación radicalmente alejada de la realidad, ya que no existe ningún estudio o investigación que haya localizado una porción no usada en nuestro cerebro.

Por otro lado, el neuromito que afirma la existencia de personas de lado derecho y de lado izquierdo, ha sido alimentado desde abundantes medios informales y no institucionales, tal y como la OECD (2007) apunta:

El cerebro está compuesto por redes neuronales, tiene áreas funcionales que interactúan entre sí y se compone de hemisferios izquierdo y derecho. Cada hemisferio se especializa más en ciertos campos que en otros. ¿Justifican estos hechos las extrañas declaraciones que se escuchan en la vida diaria, tales como: "yo soy más del lado izquierdo del cerebro" o "las mujeres tienen más desarrollado el lado derecho del cerebro"? (p. 180).

Hellige (2000) indica al respecto que la investigación procedente de la neurociencia cognitiva cuenta con décadas de investigación centradas en el estudio de las diferencias entre los hemisferios cerebrales, dando como resultados una conceptualización y un tipo de análisis que enfatizaron la visión de cada uno de los dos hemisferios como sistemas de procesamiento diferentes, atribuyéndoles unas habilidades específicas en las que radicarían esos rasgos diferenciales. Aunque no existan dudas acerca de que la lateralidad cerebral sea una propiedad fundamental del cerebro y del comportamiento humano, es necesario tener en cuenta la variedad de formas en las que los dos hemisferios interactúan, y los mecanismos biológicos que dan soporte a esa misma interacción. Geake (2008) apunta a que la línea seguida desde los contornos científicos y académicos promulga, al contrario que el neuromito, un trasvase del énfasis en la modularidad hacia uno centrado en la integración.

Rato, Abreu y Castro-Caldas (2013) se refieren a otro de los neuromitos, como una creencia asociada al tópico del multilingüismo, y en la que se sostiene que el aprendizaje simultáneo a edades tempranas de dos o más lenguas conlleva a una competición por los recursos cognitivos, resultando este hecho en la necesidad de que la primera lengua deba ser adquirida completamente antes de iniciarse el aprendizaje de una segunda. Diamond (2010), por el contrario, argumenta que los niños que aprenden una segunda lengua a edades tempranas no evidencian un descenso de competencias en las destrezas asociadas a su lengua materna. Por otro lado, en las investigaciones respecto al bilingüismo comienzan, también, a surgir evidencias que apuntan a ciertas ventajas en el desarrollo de las funciones ejecutivas en niños bilingües (Carlson y Meltzoff 2008; Kovács y Mehler, 2009) Bialystok y Viswanathan (2009), indicando que estas diferencias podrían perfilarse principalmente en los procesos de flexibilidad cognitiva asociados al cambio entre tareas y a los procesos de control inhibitorio.

En las creencias erróneas y sin rigor científico ligadas al tópico de la neuroplasticidad se refleja la influencia de la creciente toma de conciencia de la capacidad de la intervención educativa para el modelaje de las redes neurales. En este sentido destacan Posner y Rothbart (2005), quienes señalan la existencia de una relación entre el desarrollo cerebral y la educación, apuntando a los modos en los que las intervenciones educativas llevadas a cabo en la escuela pueden alterar las redes neurales asociadas a la cognición en formas que se generalizan más allá del dominio específico de la instrucción. Tal y como expresan estos autores, la adquisición de habilidades culturales mediante la educación da como resultado cambios en la plasticidad funcional que abarcan un amplio rango de redes cerebrales.Sería dentro de este mismo contexto, en el seno de las investigaciones mediante Brain Training asociadas a la neuroplasticidad, donde surgiría el cuerpo de neuromitología asociado al Brain Gym que ejemplificaría al grupo de creencias distorsionadas respecto la capacidad plástica del cerebro y a la potencialidad de determinadas tareas o ejercicios para producir mejoras en el rendimiento cognitivo.

Respecto a las creencias relacionadas con aspectos nutricionales Sigman, Peña, Goldin y Ribeiro (2014) señalan al respecto, la relevancia de la nutrición en ciertos aspectos concernientes al rendimiento académico; la influencia de sustancias como la cafeína y la glucosa en los procesos cognitivos, y en consecuencia en el aprendizaje constituyen núcleos de investigación todavía activos.

Los resultados de encuestas llevadas a cabo en diferentes países, con educadores procedentes de diversos entornos culturales, han revelado niveles altos de persistencia en creencias asociadas a estos neuromitos. Concretamente, se ha analizado la prevalencia de neuromitos entre profesorado del Reino Unido y Holanda (Dekker, Lee, Howard-Jones y Jolles, 2012); de Portugal (Rato, Abreu y Castro-Caldas, 2013); de Turkía (Karakus, Howard-Jones y Jay, 2014); de China ( Pei, Howard-Jones, Zhang, Liu y Yin, 2014); y, muy recientemente, también de futuros maestros, además de docentes, de Suiza (Tardif, Doudin, y Meylan, 2015).

En base a todo lo anterior, el objetivo general de este trabajo es el de describir el estado de prevalencia de neuromitos en futuros maestros, y sus actitudes hacia la necesidad de superación de los mismos, así como la relevancia que otorgan a la provisión de una formación que incluya las bases científicas necesarias para su disipación. Como objetivos específicos, se encuentra el de dilucidar si el nivel de formación de los estudiantes opera como factor diferencial en los resultados, tanto de conocimientos como de actitudes, constituyendo este 
mismo el principal rasgo de innovación de la presente investigación respecto de la literatura precedente, dado hasta ahora los estudios se han realizado con docentes con experiencia profesional. También se trata de aportar a los análisis transculturales una muestra tomada en nuestro país, ya que no hay estudios dentro de este ámbito geográfico.

La hipótesis de partida es que, en lo que se refiere a los conocimientos, el nivel de estudios de los participantes operará favorablemente en la disipación de los neuromitos, en concordancia con lo argumentado en la literatura precedente (Coch y Ansari, 2009; Mason, 2009; Varma, Mccandliss y Schwartz, 2008), que sitúan los procesos de actualización en la formación docente como el medio principal de erradicación de este tipo de creencias. En cuanto a las actitudes, se esperaba que resultasen más favorables también en el último curso de los estudios, debido a una toma de consciencia tras los años de formación.

\section{Método}

\section{Participantes}

En este estudio participaron voluntariamente estudiantes de ambos sexos de primero y último curso de los Grados de Educación Infantil (EI) y Educación Primaria (EP) de la Facultad de Ciencias de la Educación de la Universidade da Coruña (UDC), del curso académico 2014-15. El número total de participantes fue de 295 personas, 199 de las cuales estaban matriculadas en primer curso (85 en EI y 114 en EP) y 96 estaban matriculadas en cuarto (54 en EI y 42 en EP).

\section{Instrumentos}

1. Cuestionario de conocimientos (CC). El objetivo de este instrumento era proporcionar información acerca de la prevalencia de neuromitos en el alumnado de magisterio. Para este estudio se realizó una adaptación del cuestionario de Dekker, Lee, Howard-Jones y Jolles (2012), que había sido diseñado para su empleo en colectivos docentes del Reino Unido y Holanda. Consta de 12 enunciados a los que se debe contestar si se considera que son verdaderos o falsos, y que responden a los núcleos temáticos de aspectos específicos concernientes a resultados de la investigación sobre el cerebro (estilos de aprendizaje: ítems 1 y 10; nutrición: ítems 2, 5 y 7; $10 \%$ de uso del cerebro: ítem 3; períodos críticos para la adquisición de la lengua materna antes de iniciarse en el aprendizaje de una segunda lengua: ítem 5; plasticidad cerebral: ítems 6, 8, 11 y 12; y dominancia hemisférica: ítem 4). La puntuación de cada ítem es de 1 o 0, según la respuesta sea correcta (no creencia en neuromito) o incorrecta (creencia en neuromito).

2. Cuestionario de actitudes y atribución de relevancia (CAAR). Este instrumento es una adaptación del trabajo de Rato, Abreu y Castro-Caldas (2011), diseñado para analizar las actitudes de docentes portugueses respecto la importancia que atribuyen a la comprensión de la función cerebral en distintos ámbitos relacionados con la praxis docente. La adaptación realizada consta de siete enunciados a los que se responde mediante una escala tipo Likert de cinco puntos (siendo
$1=$ nada importante y $5=$ muy importante). También se daba la opción de contestar "no sé".

3. Cuestionario de actitudes ante propuestas orientadas a la superación de los neuromitos (CASN). Adaptado también del trabajo de Rato, Abreu y CastroCaldas (2011), que Había sido diseñado para evaluar el grado de acuerdo/desacuerdo de maestros portugueses ante distintas propuestas orientadas a la mejora de la comunicación entre el campo neurocientífico y el educativo. En la adaptación realizada para la presente investigación, estuvo formado por cinco enunciados cuya respuesta se realiza en una escala tipo Likert de cinco puntos $(1$ = nada de acuerdo y $5=$ muy de acuerdo $)$, más la opción "no sé". (Véase archivo complementario)

\section{Procedimiento}

Los datos utilizados para los análisis fueron recogidos mediante los cuestionarios, que se aplicaron de forma autoadministrada dentro de las aulas de la Facultad de Educación, en horario escolar y mediante la colaboración de los docentes responsables de las materias. Durante la realización no se produjeron rechazos y todos los presentes (a quienes se les garantizaba el total anonimato de sus respuestas) los cumplimentaron. El tiempo total empleado en responder osciló entre 5 y 19 minutos.

\section{Resultados}

Para el análisis de los datos se empleó el IBM SPSS v.22. Los resultados mostraron que la fiabilidad de las puntuaciones obtenidas con los tres cuestionarios empleados fue buena, especialmente si se tiene en cuenta el reducido número de ítems (CC: 12 ítems; $\alpha=.63$; CAAR: 7 ítems, $\alpha=.76$; CASN: 5 ítems, $\alpha=.70$ ). Es de destacar, además, que el cuestionario de conocimientos abarcaba diferentes dimensiones, lo que explicaría una consistencia interna más baja.

A continuación analizaremos por separado los resultados de la prueba de conocimientos, por un lado, y las de actitudes, por otro, para finalizar tratando de establecer relaciones entre ambas dimensiones.

\section{Prevalencia de neuromitos en futuros maestros}

Por lo que se refiere a la prueba de conocimientos, no se observaron diferencias entre las dos titulaciones de las cuales procedía la muestra $(t(293)=.958 ; p=.339)$, pero sí en relación con el curso académico $(t(293)=16.033$; $p<.001)$. Esto es: los estudiantes de primero respondieron correctamente, en promedio, un número significativamente menor de preguntas $(\bar{X}=58.37 \%$; SD $=12.88)$ que los de cuarto $(\bar{X}=83.15 \% ; S D=11.47)$. El análisis de varianza también mostró que hay diferencias significativas entre las distintas medidas de conocimiento (ítems de la prueba): $F(11,246)=334.427 ; p<.001$, lo que sería indicativo de que, como se esperaba, el nivel de conocimientos mejora al aumentar la formación académica. No obstante, analizadas por separado las diferentes cuestiones se observa que sólo se diferencian significativamente los mencionados cursos en 5 de las 12 preguntas: a) "Existen pruebas científicas que afirman que los suplementos nutritivos de ácidos grasos esenciales (omega 3 y omega6) tienen un efecto positivo en el rendimiento académico": $t(279)=3.158, p=.002)$; 
b) "Las diferencias de dominancia hemisférica (cerebro izquierdo-cerebro derecho) pueden ayudar a explicar las diferencias individual de los alumnos": $t(280)=1.378$, $p=.004 ; c)$ "Los niños deben adquirir correctamente su lengua nativa antes de se iniciará en el aprendizaje de una segunda lengua, ya que de hacer el contrario se dificultará la correcta adquisición de la primera": $t(293)=2.254, p$ $=.025 ; \mathrm{d})$ "Las tareas de prácticas continuadas en tareas de procesos mentales puede llegar a producir cambios en la estructura y forma del cerebro": $t(279)=-1.101, p=$ .019 ; y e) "Los problemas en el aprendizaje asociados la diferencias en el desarrollo de la función cerebral no pueden ser remediados mediante intervenciones educativas": $t(291)=-1.024, p=.045$.

Cabe destacar, también, que únicamente el Ítem 1 puede ser considerado como muy fácil, seguido del 12 que resultó fácil, mientras que el 10 sería muy difícil y el 2 , el 4 y el 6 difíciles, todo ello en ambos cursos. Además, el 5 y el 11 resultaron fáciles para los estudiantes de $4^{\circ}$.

\section{Actitudes}

Los resultados muestran que las actitudes de los participantes son positivas (superiores en todos los casos a punto medio de la escala de valoración), tanto en lo que se refiere a la importancia que atribuyen a la comprensión de la función cerebral en distintos ámbitos relacionados con la praxis docente, como ante las distintas propuestas orientadas a la mejora de la comunicación entre el campo neurocientífico y el educativo. Además, aunque la diferencia de medias en las puntuaciones totales no son tan grandes como en conocimientos, resultan estadísticamente significativas (CAAR: $t(293)=-2.553$; $p=.011$ y CASN: $t(293)=-3.013 ; p=.003)$, mostrando que el alumnado de cuarto tiene una actitud más positiva hacia las cuestiones abordadas.

Por otro lado, el perfil de respuestas es muy similar en ambos cursos, oscilando las puntuaciones entre 3.74 y 4.76 (sobre 5.00) para cuarto curso y entre 3.64 y 4.68 (sobre 5.00) para primero.

Analizados por separado los distintos ítems se observan diferencias estadísticamente significativas únicamente en cuatro de ellos:

- Un mayor conocimiento sobre el funcionamiento del cerebro ante el aprendizaje llevado a cabo en el aula es conveniente para: a) "Facilitar la detección tempranera de dificultades de aprendizaje": $t(284)=-2.201, p=$ .029 ; y b) "Poder dar apoyo a alumnos con discapacidad cognitiva": $t(286)=-2.236, p=.026$.

- Estas propuestas serían beneficiosas para mi formación como docente y para mi futura práctica profesional: a) "Oportunidad de asistir a conferencias o cursos que tengan como tópico central la relación entre la neurociencia y la educación": $t(288)=-2.246, p=.025$; y b) "Una colaboración más estrecha entre la escuela y la universidad": $t(290)=-2.550, p=.011$.

En relación al mayor o menos grado de aceptación de los ítems, los resultados reflejan que los ítems peor valorados son "Un mayor conocimiento sobre aspectos del cerebro y el aprendizaje es importante para el desarrollo del diseño curricular" y "Estoy de acuerdo en que la inclusión de unos principios de neurociencia educativa en mi educación sería beneficiosa para mi formación y mi futura praxis profesional", mientras que los que arrojan puntuaciones más altas son "Un mayor conocimiento sobre aspectos del cerebro y el aprendizaje es importante para poder dar apoyo a alumnos con discapacidad cognitiva" y "Estoy de acuerdo en que una colaboración más estrecha entre la escuela y la universidad sería beneficiosa para mi formación y mi futura praxis profesional".

\section{Relación entre conocimientos y actitudes}

Analizada la relación entre las distintas creencias sobre neuromitos y las actitudes, los resultados muestran que sólo una opinión se relaciona significativamente con el nivel de conocimientos: la relativa a la "Inclusión de unos principios básicos de neurociencia educativa en la formación" ( $r=.144 ; p=.016)$, en la cual no había diferencias entre primero y cuarto curso.

Visto desde el otro ángulo, los conocimientos que se relacionan significativamente con las actitudes globales son, por un lado, "Las tareas consistentes en series cortas de ejercicios de coordinación pueden revertir en mejoras en la integración de los hemisferios derecho e izquierdo", que tiene una correlación negativa tanto con la importancia concedida a un mayor conocimiento del cerebro como a las propuestas que podrían beneficiar la futura práctica docente $(r=-.142 ; p=.017$ y $r=-.195 ; p$ $=.001$, respectivamente). En cambio "Los problemas en el aprendizaje asociados la diferencias en el desarrollo de la función cerebral no pueden ser remediados mediante intervenciones educativas" correlaciona positivamente, pero sólo con lo que tiene que ver con las ventajas de un mayor conocimiento sobre el funcionamiento del cerebro $(r=.149 ; p=.011)$, mientras que "Las tareas de prácticas continuadas en tareas de procesos mentales puede llegar a producir cambios en la estructura y forma del cerebro" correlaciona con la actitud ante propuestas orientadas a la mejora de la comunicación entre el campo neurocientífico y el educativo $(r=.117 ; p=.050)$.

\section{Discusión}

De acuerdo con los datos disponibles, la muestra del presente trabajo alcanzó al $63 \%$ de los matriculados por primera vez en EI y al $85 \%$ de los de EP. Respecto a $4^{\circ}$ curso, y tomando en cuenta la última matrícula publicada (curso 2013-14), alcanzó al 46 \% en EP y al $61 \%$ en EI. Sabiendo que los cuestionarios se pasaron exclusivamente a quienes asistían a clases, a la vista de los porcetajes, se podría concluir que la muestra es amplia, contándose con más participación en EP.

Empleando en el presente trabajo una muestra íntegramente formada por participantes en proceso de instrucción formal habilitador para la profesión educativa, se pretendía dilucidar si la formación de los educadores puede operar como eje disipador de las creencias erróneas asociadas a los distintos neuromitos. Tal y como los resultados reflejan, se encuentran unos niveles generales de descenso del nivel de creencias relacionadas con la neuromitología entre los participantes a medida que su proceso de instrucción avanza. Además, realizando un análisis comparativo entre este estudio y trabajos precedentes, los resultados arrojan balances positivos. 
Así, a la vista de los resultados, sería posible afirmar que algunos neuromitos se encuentran prácticamente erradicados en toda la muestra empleada en este trabajo, en concreto aquellos correspondientes a "Cada alumno posee una preferencia respecto a las modalidades en las que les presentamos la información (auditiva, visual, táctil..." y a "Los problemas en el aprendizaje asociados la diferencias en el desarrollo de la función cerebral no pueden ser remediados mediante intervenciones educativas", reflejándose unos porcentajes de aciertos del $97.29 \%$ y del 85.05 , respectivamente. Esto contrasta con los resultados de estudios precedentes, encontrándose marcadas diferencias con la muestra de Turquía donde aflora un nivel de prevalencia para ese tipo de creencias de porcentajes de aciertos de solo un $34.89 \%$ para el primer ítem y un $7.91 \%$ para el segundo.

También cabe señalar que en la muestra de nuestro país se observan ciertos niveles de irregularidad, que vienen reflejados por unos porcentajes de aciertos más bajos en relación al resto de participantes en "Las diferencias de dominancia hemisférica (cerebro izquierdo-cerebro derecho) pueden ayudar a explicar las diferencias individual de los alumnos" y "Las tareas consistentes en series cortas de ejercicios de coordinación pueden revertir en mejoras en la integración de los hemisferios derecho e izquierdo", con un $24.41 \%$ y un $16.95 \%$, respectivamente.

Por el contrario, en el resto de datos analizados se observan niveles de aciertos más altos para nuestros participantes, en comparación con los grupos de referencia, en el ítem "Sólo usamos el 10\% de nuestro cerebro", y en "A los niños les resultará más difícil atender después de tomar bebidas azucaradas o golosinas" (excepto en lo que se refiere a los Países Bajos y el Reino Unido).

Respecto al resto de ítems que el cuestionario de conocimientos comparte con estudios precedentes en Reino Unido y Holanda (Howard-Jones, P. A., 2014), los niveles en porcentajes de aciertos para los futuros maestros que estudian en la UDC son, en 2 de los 5 ítems, superiores a los de los participantes de ambos países: para el ítem "Existen pruebas científicas que afirman que los suplementos nutritivos de ácidos grasos esenciales (omega 3 y omega 6) tienen un efecto positivo en el rendimiento académico" el porcentaje de aciertos es de un $25.17 \%$ frente al $12 \%$ de la muestra tomada en Reino Unido y al $16 \%$ de la muestra tomada en Holanda; en el ítem Las bebidas con cafeína tomadas de forma regular reducen el estado de alerta" el porcentaje de aciertos en nuestro país es de un $50.62 \%$ mientras que en los docentes de Reino Unido y Holanda es de un $39 \%$ y un $41 \%$ respectivamente. Los resultados solo se muestran inferiores a ambos grupos de referencia en el ítem "Las prácticas continuadas en tareas de procesos mentales pueden llegar a producir cambios en la estructura y forma del cerebro", observándose en la muestra de Reino Unido un porcentaje de aciertos del $69 \%$ y de un $58 \%$ para la muestra de Holanda, frente al 55.82 obtenido en el presente estudio.

En el caso de las actitudes, los resultados de este trabajo siguen la tendencia marcada por estudios precedentes, en los que las posturas ante las distintas propuestas presentadas se perfilan como marcadamente favorables. Así, contrastando con una investigación similar llevada a cabo con 627 maestros portugueses con ejercicio profesional, desde Educación Infantil hasta Educación Secundaria (Rato, Abreu y Castro-Caldas, 2011), se observa que las actitudes respecto a la relevancia de la neurociencia en aspectos que tienen que ver con el abordaje de las necesidades educativas relacionadas con aspectos cognitivos, reflejan los mayores niveles de aceptación en ambos estudios, encontrándose una coincidencia en los tres ítems mejor valorados por ambas muestras. Concretamente, el ítem que se refiere a la relevancia de la neurociencia para el afrontamiento de las necesidades educativas asociadas a dificultades cognitivas, es el de mayor puntuación en los dos trabajos, con una valoración de 4.61 sobre 5 en la muestra de la UDC y unos niveles de aceptación del 93\% en la muestra portuguesa. En el ítem que se refiere a que "Un mayor conocimiento sobre el funcionamiento del cerebro ante el aprendizaje llevado a cabo en el aula es importante para la detección temprana de dificultades de aprendizaje", las similitudes se mantienen, constituyendo el segundo mejor valorado (4.61 sobre 5 para UDC y un $91 \%$ para los maestros portugueses). En cuanto al tercero mejor valorado (importancia de tener un mayor conocimiento del cerebro para la elección de los métodos de enseñanza más adecuados), el paralelismo entre ambos estudios se mantiene, con una puntuación de de 4.27 sobre 5 para la muestra de futuros maestros de nuestro estudio y un $87 \%$ de aceptación en el profesorado portugués.

En el ámbito de las propuestas sobre el asentamiento de unos mejores canales de comunicación entre el área de la neurociencia y el de la educación, ambas muestras otorgan niveles de relevancia altos al ítem concerniente a la creación de escuelas de investigación donde los profesionales educativos y los de las ciencias cognitivas puedan trabajar aunando esfuerzos dentro del campo de la investigación educativa.

\section{Conclusiones}

Aunque los resultados favorables que emergen de la recogida de datos llevada a cabo en este estudio proceden de una muestra restringida en cuanto al ámbito geográfico, los trabajos publicados suelen tener también muestras bastante homogéneas e, incluso, bastante más pequeñas que la empleada aquí. Por otro lado, nuestra investigación constituye uno de los primeros intentos por esclarecer el debate en cuanto a la necesidad de actualización en la formación de los futuros maestros, en la que se proporcionen unos contenidos o alfabetización básica en principios de neurociencia educativa. Se concluye, por tanto, que la formación recibida por los futuros docentes ha respondido a esa necesidad de actualización, contribuyendo al conocimiento en aquellos núcleos temáticos considerados clave para evitar la construcción de neuromitos. Sin embargo, no se puede obviar el hecho de que, en algunas creencias concretas, los niveles de persistencia de neuromitología presentan niveles elevados, hecho que concuerda con la literatura precedente, situando al colectivo docente como uno de los principales receptores de este tipo de creencias. 


\section{Referencias}

Ansari, D., y Coch, D. (2006). Bridges over troubled waters: Education and cognitive neuroscience. Trends in Cognitive Sciences, 10, 146-151. http://dx.doi.org/ 10.1023/A:1022145812276

Bialystok, E., \& Viswanathan, M. (2009). Components of executive control with advantages for bilingual children in two cultures. Cognition, 112, 494500. http://dx.doi.org/10.1016/j.cognition.2009.06.014

Calvert, G. A., Campbell, R., \& Brammer, M. J. (2000). Evidence from functional magnetic resonance imaging of crossmodal binding in the human heteromodal cortex. Current biology, 10, 649-657. http://dx.doi.org/ 10.1016/S0960-9822(00)00513-3

Carlson, S. M., \& Meltzoff, A. N. (2008). Bilingual experience and executive functioning in young children. Developmental science, 11, 282-298. http:// dx.doi.org/10.1111/j.1467-7687.2008.00675.x

Coch, D., y Ansari, D. (2009). Thinking about mechanisms is crucial to connecting neuroscience and education. Cortex, 45, 546-547. http://dx.doi.org/10. 1016/j.cortex.2008.06.001

Crockard, A. (1996). Confessions of a brain surgeon. New Scientist, 2061, 2068.

Dekker, S., Lee, N., Howard-Jones, P., y Jelle, J. (2012). Neuromyths in education: Prevalence and predictors of misconceptions among teachers. Frontiers in Psychology, 3. http://dx.doi.org/10.3389/fpsyg.2012. 00429

Diamond, J. (2010). The benefits of multilingualism. Science, 330, 332-333. http://dx.doi.org/10.1126/ science. 1195067

Geake, J. (2008). Neuromythologies in education. Educational Research, 50, 123-133. http://dx.doi.org/ $10.1080 / 00131880802082518$

Hellige, J. (2000). All the King's horses and the king's men: Putting the brain back together again. Brain and Cognition, 42, 7-9. http://dx.doi.org/10.1006/brcg. 1999.1146

Howard-Jones, P. A. (2014). Neuroscience and education: myths and messages. Nature Reviews Neuroscience, 15, 817-824. http://dx.doi.org/10.1038/ nrn3817

Karakus, O., Howard-Jones, P. A., \& Jay, T. (2015). Primary and Secondary School Teachers' Knowledge and Misconceptions about the Brain in Turkey. Procedia-Social and Behavioral Sciences, 174, 19331940. http://dx.doi.org/10.1016/j.sbspro.2015. 01.858

Kovács, Á. M., \& Mehler, J. (2009). Cognitive gains in 7-month-old bilingual infants. Proceedings of the National Academy of Sciences, 106, 65566560. http://dx.doi.org/10.1073/pnas.0811323106

Mason. L. (2009). Briding neuroscience and education: A two-way path is possible. Cortex, 45, 548549. http://dx.doi.org/10.1007/s10648-012-9207-x

OCDE (2002). Understanding the brain: towards a new learning science. Paris: OCDE

OCDE (2007). Understanding the brain: towards a new learning science. Paris: OCDE

Pei, X., Howard-Jones, P. A., Zhang, S., Liu, X., \& Jin, Y. (2015). Teachers' Understanding about the Brain in East China. Procedia-Social and Behavioral Sciences, 174, 3681-3688. http://dx.doi.org/10.1016/j.sbspro. 2015.01.1091

Posner, M. I., y Rothbart, M. K. (2005). Influencing brain networks: implications for education. Trends in cognitive sciences, 9, 99-103. http://dx.doi.org/10. 1016/j.tics.2005.01.007

Rato, J., Abreu, A., y Castro-Caldas, A. (2011). Achieving a successful relationship between neuroscience and education: The views of Portuguese teachers. Procedia-Social and Behavioral Sciences, 29, 879-884. http://dx.doi.org/10.1016/j.sbspro.2011. 11.317

Rato, J., Abreu, A., y Castro-Caldas, A. (2013). Neuromyths in Education: What is fact and what is fiction for Portuguese teachers. Educational Research, 55, 441-453. http://dx.doi.org/10.1080/00131881. 2013.844947

Takekuchi, H., Sekiguchi, A ., Yokokama, S., Yomogida, Y., Komuro, N., Yamanouchi, T., Suzuki, S., y Kawashima, R.J. (2010). Training of working memory impacts structural connectivity. Journal of Neuroscience. 30, 3297-3303. http://dx.doi.org/10. 1523/JNEUROSCI.4611-09.2010

Varma, S., McCandliss, B. D., \& Schwartz, D. L. (2008). Scientific and pragmatic challenges for bridging education and neuroscience. Educational Researcher, 37, 140-152. http://dx.doi.org/10.1007/s10648-0129207-x 